\title{
Virtual Reality Rehabilitation and Exergames-Physical and Psychological Impact on Fall Prevention among the Elderly-A Literature Review
}

\author{
Joanna Piech ${ }^{1,2, *(1)}$ and Krzysztof Czernicki ${ }^{2}$ (i) \\ 1 Faculty of Health Sciences, Jagiellonian University Medical College, 33-332 Krakow, Poland \\ 2 Department of Rehabilitation, Lesser Poland Orthopedic and Rehabilitation Hospital, 30-224 Krakow, Poland; \\ kczernicki@gmail.com \\ * Correspondence: joanna.byra@doctoral.uj.edu.pl
}

check for

updates

Citation: Piech, J.; Czernicki, K. Virtual Reality Rehabilitation and Exergames-Physical and

Psychological Impact on Fall

Prevention among the Elderly-A Literature Review. Appl. Sci. 2021, 11, 4098. https://doi.org/10.3390/ app11094098

Academic Editors: Youngho Lee and Elena Amaricai

Received: 24 March 2021

Accepted: 27 April 2021

Published: 30 April 2021

Publisher's Note: MDPI stays neutral with regard to jurisdictional claims in published maps and institutional affiliations.

Copyright: (C) 2021 by the authors. Licensee MDPI, Basel, Switzerland. This article is an open access article distributed under the terms and conditions of the Creative Commons Attribution (CC BY) license (https:/ / creativecommons.org/licenses/by/ $4.0 /)$.

\begin{abstract}
The present review is aimed at the effectiveness of virtual reality (VR) and exergames in the prevention of falls among the elderly. Falls become a significant problem in the aging population and lead to psychological, social, and physical impairment. Prevention of falls is crucial to the well-being of the elderly population and is one of the challenges of contemporary rehabilitation. Recently, in view of the threat of the SARS-CoV-2 pandemic, contactless methods of rehabilitation, including telerehabilitation, appear as valuable rehabilitation tools. This review is based on the PRISMA guidelines and was carried out in five databases: PubMed, Medline, Web of Science, Scopus, and PEDro. Twenty-one randomized controlled trials, focused on the application of VR and exergames in the prevention of falls, were included. This review suggests that VR training in rehabilitation appears to be a promising complement to traditional techniques of physiotherapy to improve specific physical outcomes. VR and exergames could be considered as a complement of standard physiotherapy and its possible continuation at home for elderly. However, further high-quality studies, with carefully designed protocols and proper blinding, are needed.
\end{abstract}

Keywords: virtual reality; exergames; falls prevention; rehabilitation; elderly

\section{Introduction}

Falls become a significant problem in the aging population. Falls are prominent factors of accidental or unintentional injuries implied by external causes $[1,2]$. The incidence of falls amongst elderly people, according to the World Health Organization, varies from $28-35 \%$ of individuals aged 65 and over, increasing to $32-42 \%$ for those over 70 years of age [1]. Falls result in the fear of falling, loss of independence, institutionalization, and eventually death [3]. Strategies to prevent falls are crucial to the well-being of the elderly population, and rehabilitation plays an important role among them.

Standard rehabilitation protocol focused on the prevention of falls comprises kinesiotherapy (exercises) and education. Exercises should include training of body balance, coordination, gait, changing the body position, minimizing the possibility of an injury during a fall, and techniques of getting up properly after a fall. Exercises are effective in reducing the risk of falling [4].

Advances in medical technology resulted in the popularization of computer-assisted interventions in rehabilitation treatment. New technologies in rehabilitation comprise of a variety of biofeedback implementations into hardware platforms, motion capture systems augmented by biofeedback, augmented reality (AR) systems, and virtual reality (VR) systems. Technological appliances vary from adoptions of popular gaming hardware platforms to specialized systems with dedicated hardware and software. Software protocols, presuming adequate hardware implementation, can be integrated into a complete computer game, which may result in better adherence. An application of virtual reality 
techniques in exergames can result in even better immersion into "digital worlds" and leads to the proposal of reframing the interpretation of virtual reality into two components: virtual reality experience (VRE) and virtual reality systems (VRS) [5]. The virtual reality experience comprises at least four interrelated contributors: immersion, interaction, sensorimotor contingencies, and illusions. Virtual reality systems refer to physical solutions to generate virtual reality experience.

Among the contributors of VRE, the effectiveness of interaction bonds them all together, eventually resulting in illusion. The interaction in VRE starts with visual exploration of the space, which can be provided by simple display, stereoscopic display, head-mounted display (HMD) with head tracker or more advanced motion-tracking systems. The experience can be further augmented by spatial sound effects, haptic data gloves etc. Next level interaction can be provided by means of brain and other bodily inputs, leading to so-called neurogames, which use brain or muscle signals to control basic interaction features [5]. Such implementations require the application of brain-computer interfaces (BCI), which are capable to non-invasively collect and further interpret collected brain signals. Regarding the input concepts, one has to distinguish augmented reality, which combines virtual and real world, and virtual reality, which substitutes real world with multiple sensorial channels, leading to more or less complete artificial stimulation, creating non-immersive or immersive VR. BCIs can be used both in AR and VR applications [6,7]. In view of rapid technology advancements, solutions in the form of small brain implants are inevitably coming, forerun by experimental devices like scalable high-bandwidth brain-machine interface system [8].

Exergames are defined as the activity of playing video games that involve physical exertion. Interventions making use of exergames have a favorable effect on both motor and cognitive functions $[9,10]$. Exergames have promised a form of intervention, which improves physical function in older adults, while endangering few adverse effects [11].

Exergaming, as an interactive, entertaining and engaging form of exercise, may help to overcome traditional exercise barriers and improve adherence in the case of older adults. Exergames provide therapeutic applications for balance recovery and functional mobility [12]. They can improve cognitive and physical functions on the basis of increased sensorial flow physical effort [9]. Recent studies augment benefits of VR in particular functions, like geographical distance estimations in relation to chosen locomotion techniques in virtual environment [13].

Exergaming, especially in older people, may have its drawbacks. There are at least two groups of factors, which have to be taken into account: the reluctance in accepting new technologies by elder people and the presence of adverse effects related directly to VR applications.

The attitudes among elderly people toward using VR are still unclear, however, a recent study demonstrates the use and acceptance towards the adoption of VR among the older population [14].

The adverse effects of VR could be identified as non-related to intervention and related to intervention [11]. The former includes all medical events not related to therapeutic sessions, e.g., falls between sessions. The latter includes adverse events like injuries, falls or medical problems, and side effects during exercises, like tiredness, muscular pain, discomfort, and motion sickness. Visual and cognitive aftereffects, chiefly related to HMD applications, are defined as VR sickness [15]. VR sickness may lead to symptoms of nausea, eye strain, headaches, dizziness, and disorientation that last beyond the exposure period $[16,17]$. Based on recent research, the VR sickness seems to last for a relatively short period of time after training [18].

While exergaming has been proven as an effective standalone intervention, the evidence of its possible prevalence over standard rehabilitation protocols is scarce [19]. 
Recently, in view of the threat of the SARS-CoV-2 pandemic, contactless methods of rehabilitation, including telerehabilitation, appear as valuable rehabilitation tools [20]. Rehabilitation is an important and necessary part of the treatment and prevention of many diseases. Therefore, its safe continuation during the pandemic prefers remote methods. Nowadays, healthcare has to deal with the challenges of providing patient care using modern technologies [21]. Their employment involves economic costs, employee training, and adaptation of the equipment to the treatment goals and the age profile groups of patients. The use of new technologies in rehabilitation carries the risk of exclusion of elderly people. Therefore, knowledge about the possibilities of the use and the adaptation of virtual reality in such a case appears to be particularly important.

The influence of interventions based on new technologies on the course of rehabilitation, regarding their hypothetical advantage over standard rehabilitation, still remains an open question. This statement is also valid in the challenging problem of the prevention of falls among elderly people.

The present study was conducted to determine, based on the current literature review, whether the virtual reality and exergames employed in rehabilitation significantly reduce the incidence of falls among elderly patients. An additional aim of this review was to assess the impact of this type of intervention on patients' cognitive functions, quality of life, adherence, usability, and enjoyment of exergames in the process of rehabilitation.

\section{Materials and Methods}

\subsection{Literature Search Strategy}

The aim of the literature review was the evaluation of the effectiveness of virtual reality rehabilitation among elderly patients in the prevention of fall risk. The methodology was based on the Preferred Reporting Items for Systematic Reviews and Meta-Analyses (PRISMA) guidelines and conducted following the PRISMA checklist [22]. A literature review was carried out in six databases: Medline, PubMed, Scopus, Embase, Web of Sciences, and PEDro.

The tool used to create the research objective and search strategy was PICOT(T) [23].

- Population-healthy elderly

- Intervention-virtual reality, exergames rehabilitation

- Comparison - any intervention (standard rehabilitation, physical exercises, daily living activities) or no intervention

- Outcomes-fall risk and prevention, body balance, walking, functional improvement, pain, cognition, quality of life

- $\quad$ Time-last 5 years (January 2015-December 2020)

- (Type of study) - randomized controlled trials

The primary search keywords were "elderly", "virtual reality", "exergames" "rehabilitation", "falls", and their synonyms. Keywords were combined using the Boolean operators "AND" and "OR". The search strategy was flexible and adapted to the database search engines. For the PubMed, we used the following search scheme: ((((virtual reality OR augmented reality OR video games OR game* OR computer games OR wii OR exergames OR vr))) AND (((rehabilitation OR exercise * OR physical therapy OR physiotherapy OR physical activity OR activity OR training))) AND ((elderly OR aged OR older OR elder OR geriatric OR elderly people OR old people OR senior))) AND (((fall prevention OR preventing falls OR prevent falls OR falls OR falling))) AND (Randomized Controlled Trial[ptyp]). Each search strategy was checked by two authors.

\subsection{Study Selection and Data Extra Action}

Firstly, two authors created the criteria for eligibility. When both authors had accepted the search strategy, one of them searched the databases (last search: January 2021). Then, two researchers reviewed all the studies (title, abstract, and full text) independently, using an automation tool-Rayyan [24]. The results were compared and all occurring conflicts at every stage of study selection and data extraction were solved through discussion. 
Articles meeting the following criteria were included in the review: (1) published in the last 5 years (January 2015-December 2020); (2) English language; (3) randomized controlled trials (RCT); and (4) targeted for the healthy elderly, rehabilitation, and exergames. Studies were excluded due to: (1) publishing before 2015; (2) writing in a language other than English; (3) reports from books, case reports, observational, as well as interventional (non-randomized) clinical trials, and protocols of RCTs; and (4) do not concern the elderly, rehabilitation, and exergames.

The data was extracted from articles by two authors. Quantitative variables were presented as mean, minimum, and maximum values. Qualitative variables were described as percentage frequencies.

\subsection{Quality Assessment}

The methodological quality was assessed using the PEDro scale, which is considered relevant for RCTs related to physical therapy $[25,26]$. The scale evaluates internal validity of the trials (criteria 2-9) and information on statistics (criteria 10-11). Criterion 1 relates to external validity but is not considered when calculating the final score. The PEDro scale is only used to evaluate the research methodology. Therefore, the PEDro results do not provide evidence of clinical utility and cannot assess the effectiveness of an intervention in clinical practice [27].

The methodological evaluation of the included articles that were available in the PEDro database was taken from the website https: / /www.pedro.org.au/ (accessed on 5 January 2021).The remaining three articles [28-30] were rated on the 11-points PEDro scale by two authors independently, then the results were compared, and potential differences were resolved during the discussion. PEDros' items were scored 0 if not reported or unclear, 1 when reported adequate. Research that received from 1-3 points was rated as poor quality, from 4-6 points was moderate quality, and above 7 points was assessed as high quality.

\section{Results}

\subsection{Searching Results}

The selection of articles was carried out in accordance with PRISMA guidelines. After searching the databases and removing duplicates, 682 articles were screened by titles and abstracts. As a result of the selection, 114 articles were included in the analysis of the full texts. After the full-text selection, 93 publications were rejected and 21 studies were included in the qualitative synthesis. For details on the selection of studies and the reasons for their rejection, see Figure 1. PRISMA flowchart.

\subsection{Characteristics of Included Studies and Participants}

Twenty-one RCTs were included in the analysis, involving a total of 1557 participants who were randomized to the study or control group, respectively. These were the healthy elderly with a mean age of 75.7 years who were examined for the risk of falls. Among the patients, the majority were women (female-to-male ratio of 1.6:1). Participants were recruited mainly from seniors residing in retirement homes and among rehabilitation ward inpatients. Post-intervention follow-up was used in 6 out of 21 studies and ranged from 6 weeks [31] to 1 year [32]. 

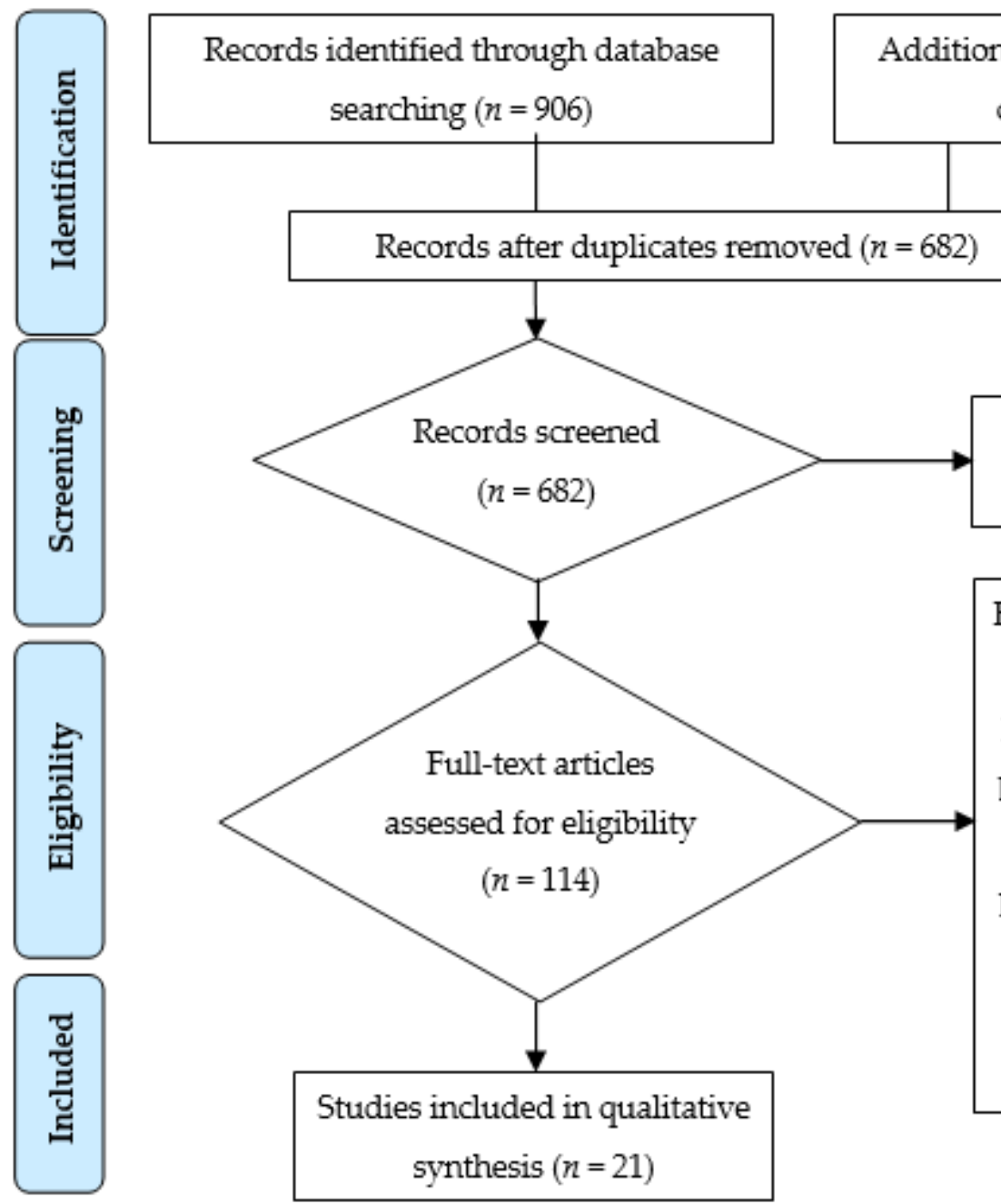

Records excluded

$(n=568)$

Full-text articles excluded, with reasons $(n=93)$

Incorrect study design $(n=15)$

Do not concern healthy elderly

$(n=31)$

Do not apply VR or exergames

$$
(n=23)
$$

Do not concern study aim

$(n=24)$

Figure 1. Flowchart of the article selection process (2009 Preferred Reporting Items for Systematic Reviews and MetaAnalyses (PRISMA) flow diagram).

\subsection{Technology of Intervention}

The main tool used for rehabilitation was virtual reality games. The exercise program was aimed at increasing muscle strength and improving body balance. In most cases, the training was based on Nintendo Wii balance boards [33-37], biofeedback [32,38], VR dancing [32,39], and exergames obtained for various physical exercises [28,39-43]. In addition to physical exercises, some studies also used exercises aimed at cognitive function and memory $[32,39,40,43]$. The experimental group received training under the supervision of a physiotherapist or trainer in 13/21 of the included studies. Unsupervised training focused on interventions at the patient's home $[30,39,42]$. The control group also consisted of the elderly who were usually treated with standard physiotherapy including balance exercises or preventive leaflets. The duration of the intervention ranged from a minimum of 10 days [44] to a maximum of 6 months [32], an average of 8.9 weeks. It should be noted that in 8 out of the 21 studies, an intervention in the experimental group was based on VR training and the same standard intervention as in the control group (e.g., exercises or education leaflets). Other research works compared VR training standalone in the experimental group and usual care in the control group. Table 1 presents more information on the intervention use. 
Table 1. Characteristics of intervention used in included studies.

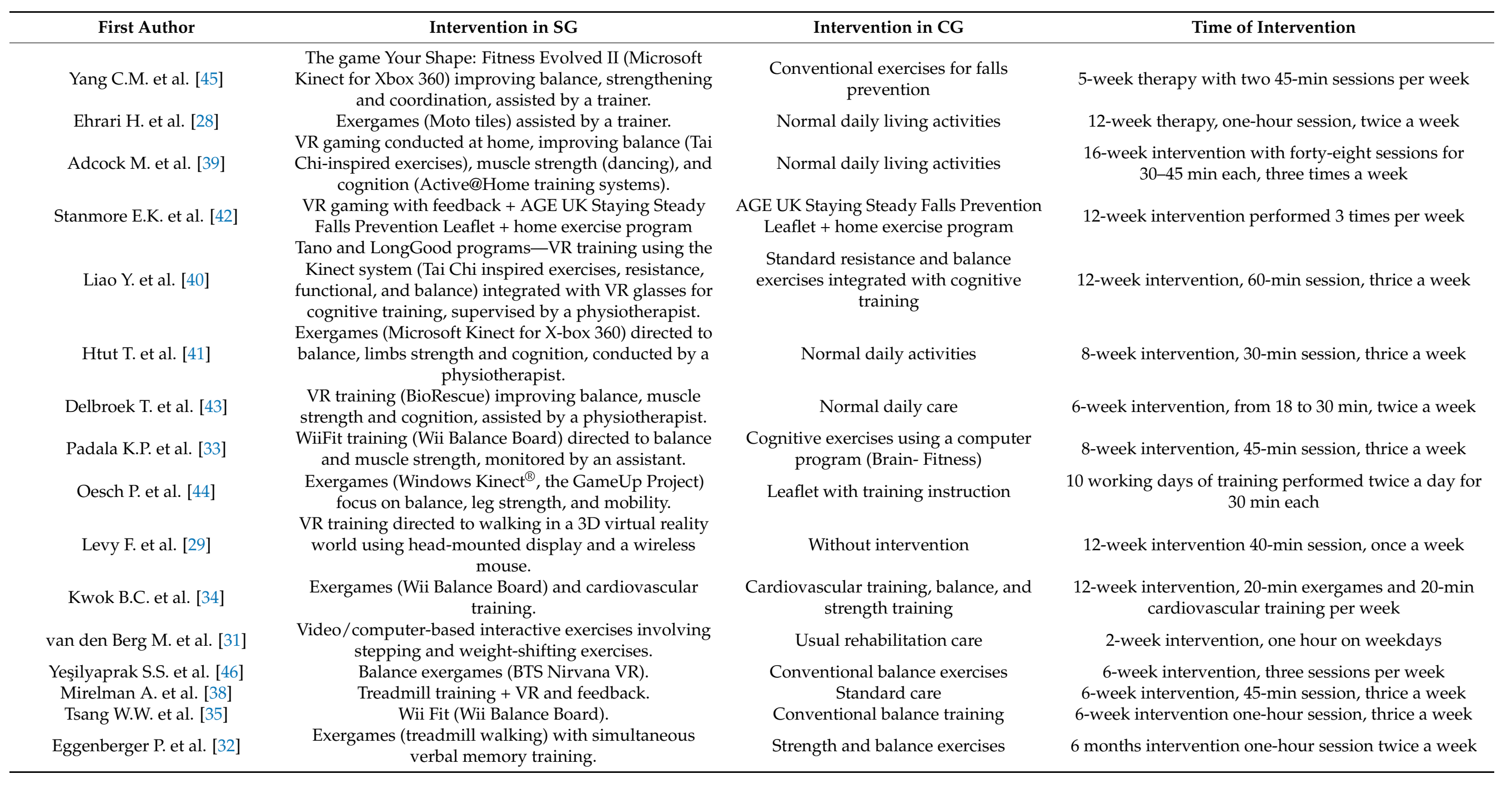


Table 1. Cont.

\section{First Author}

Gschwind Y.J. et al. [47]

Gschwind Y.J. et al. [30]

Jung D.I. et al. [37]

Park J. et al. [48]

Whyatt et. al. [36]

\section{Intervention in SG}

Balance exergames, strength exercises, and education

$$
\text { booklet. }
$$

Exergames (Microsoft Kinect for X-box 360) and step mat training, unsupervised home training.

Exergames (Nintendo Wii Sports) and lumbar stabilization exercises.

Exergame (3D VR Kayak program) and conventional exercise program.

Balance exergames (Wii Balance Board).

\section{Intervention in CG}

\section{Time of Intervention}

Education booklet about evidence-based health and fall prevention advice Educational booklet about

evidence-based health and fall prevention advice

Without intervention

Conventional exercise program Normal activity (self-recorded diary) 16-week intervention 120-min a week

16-week intervention

8-week intervention 30-min session, twice a week

6-week intervention, twice a week

5 -week intervention, twice a week 


\subsection{The Effectiveness of Virtual Reality and Exergames}

\subsubsection{Quantitative Outcomes}

Quantitative outcomes were assessed as the physical performance of patients (Table 2) and mainly contain the following activities: balance, gait ability, risk of falling, and muscle strength. The main tools used to evaluate the above variables were the Berg Balance Scale in 8/21 studies, the Timed Up and Go Test and its variants in 10/21 studies, the Falls Efficacy Scale-International and its variations (FES-I) in 6/21 studies, and the 10-to-2-min march test in 4/21 studies. Short Physical Performance Battery (SPPB) was used in 6/21 studies to evaluate physical activity and risk of disability in activities of daily living.

The assessment of the impact of VR rehabilitation and exergames on physical function among the elderly varies in the analyzed studies. In 11 out of 21 studies, there was a significantly greater improvement among assessed specific outcomes for VR training than conventional therapy. Moreover, 8 of the 21 pieces of research assessed VR-based intervention as being effective as traditional treatment. On the other hand, 2 of the 21 analyzed studies $[39,44]$ indicate no special benefits for VR intervention in comparison to standard therapy. These results indicate that new technologies used in rehabilitation could be equally effective in the improvement of specific physical function among elderly patients. However, the diversity in technologies used and research tools among included studies makes impossible to draw a clear conclusion that any intervention based on VR will be effective in the elderly in terms of improving physical function. Additionally, in the assessment of statistical significance of the results, many studies used only $p$-value to misinterpret the effectiveness of the application of the intervention. However, $p$-value only shows that an effect exists and does not reveal the size of the effect. In quantitative research, both $p$-value and size effect should be reported. Therefore, the lack of information on the size effect does not allow a full assessment of the effectiveness of the intervention. 
Table 2. Quantitative Outcomes.

First Author

\section{Yang C.M. et al. [45]}

Ehrari H. et al. [28]

Adcock M. et al. [39]

Stanmore E.K. et al. [42]

Liao Y. et al. [40]

Htut T. et al. [41]

Delbroek T. et al. [43]

\section{Outcomes}

Leg strength and endurance, balance, joint pain

Balance, leg strength and endurance, aerobic capacity, daily number of steps

Gait analysis, single task walking and dual task walking, balance, leg strength and endurance, brain plasticity/brain volume

Balance, mobility, physical activity, falls

Gait analysis

Balance, risk of falling, muscle strength, risk of falling, perception of exercise effort
BBS, TUG, TUG-cog, 5TSTS, HGS, FES-I, Borg CR-10
Balance, gait, dual-task performance (cognitive-motor)

Research Tools

30-s CST, TUG, FRT, OLST with eye open and closed, VAS

BBS, 30-s CST, 6MWT, SENS motion-sensors $24 / 7$

Step length, step speed, step time, MTC by the Physilog ${ }^{\circledR} 5$, SPPB, SFT, MRI and VBM

BBS, TUG, PASE, Short FES-I, FRAT including VAS pain and VAS fatigue

The GAIT Up system

\section{Results}

Both interventions improved balance, but only VR training improved FRT $(p=0.021)$ in comparison with controls.

Both interventions improved physical outcomes, but there was no significant difference between the SG and CG in measured outcomes.

No significant improvement between the SG and CG in physical parameters and brain plasticity/volume $(p>0.05)$ with the exception of an interaction effect in the $30 \mathrm{~s}$ chair rises test (component of SFT) $\left[\mathrm{F}(1)=5.076, p=0.024, \eta^{2}=0.01\right]$.

Significant positive impact on balance (BBS: $6.295 \%$ CI 2.4 to 10.0 ; $p=0.003, \mathrm{IRR}=0.08)$, pain (VAS: $-12.1,95 \% \mathrm{CI}-22.3$ to $-1.8, p=0.024)$ fear of falling (FES-I: adjusted mean difference $=-2.7,95 \% \mathrm{CI}-4.5$ to $-0.8, p=0.007$ ), and future falls prevention (IRR $=0.3195 \%$ CI 0.16 to 0.62 $p=0.001)$ in the SG compared to CG.

Both interventions presented significant improvements in single and motor gait performance and inhibition, but only VR improved cognitive dual-task gait $(p=0.003)$

All interventions improved physical strength, cognition, falls prevention, and body balance, but VR had a higher impact on physical as well as cognition performance. BBS: all interventions were significantly $(p<0.001)$ greater than the $C G$ with the effect size of $P E=1.59, \mathrm{VR}=1.65$, and $\mathrm{BE}=1.52$. TUG: $\mathrm{PE}$ group had a significant decrease in time compared with VR $(p=0.004$, effect size $=0.93)$ and BE $(p=0.012$, effect size $=0.75)$. 5TSTS: all interventions were significantly (PE $p<0.001$; VR $p<0.001$; and

$\mathrm{BE} p=0.036)$ compared with CG with the effect size of $\mathrm{PE}=1.62$,

$\mathrm{VR}=1.42$, and $\mathrm{BE}=0.60$. HGS was significantly greater in PE (Left $p<0.005$; Right $p<0.005$ ) and VR (Left $p<0.005$; Right $p<0.005$ ) than the CG with the effect size for PE Left $=0.84$ and Right $=0.90$, and for VR was Left $=0.88$ and Right $=0.83$. FES-I: VR was significantly lower than PE $(p=0.036$, effect size $=0.58), \mathrm{BE}(p=0.011$, effect size $=0.77)$, and CG $(p<0.001$, effect size $=1.24)$.

No changes in the Tinetti-POMA. VR training is more effective than conventional treatment only in dynamic balance in single task walking-iTUG improved after 6 weeks training in the IG (17.2 s vs. $15.8 \mathrm{~s}, p=0.02)$. 
Table 2. Cont.

\begin{tabular}{|c|c|c|c|}
\hline First Author & Outcomes & Research Tools & Results \\
\hline Padala K.P. et al. [33] & Balance, fear of falling & $\mathrm{BBS}, \mathrm{ABC}$ & $\begin{array}{l}\text { The Wii Fit training was effective in improving balance in elderly (BBS at } \\
4 \text { weeks: } 2.7(1.2-4.2, p=0.001 \text {, and BBS at } 8 \text { weeks: } 4.1(2.6-5.7, p<0.001)\end{array}$ \\
\hline Oesch P. et al. [44] & Balance & Tri-axial ActiGraph accelerometer & $\begin{array}{c}\text { No benefits were found for exergames compared with conventional } \\
\text { exercises regarding balance skills. }\end{array}$ \\
\hline Levy F. et al. [29] & $\begin{array}{l}\text { Fear of falling, functional } \\
\text { impairment }\end{array}$ & FFM, SDS & $\begin{array}{c}\text { There was a statistically significant difference between SG and CG for } \\
\text { reducing fear of falling }(p=0.007) \text {. }\end{array}$ \\
\hline Kwok B.C. et al. [34] & $\begin{array}{l}\text { Fear of falling, knee strength, } \\
\text { balance, risk of falling, physical } \\
\text { function, falls rate }\end{array}$ & $\begin{array}{l}\text { MFES, KES, TUG, HGS, 6MWT, } \\
\text { NCWT, SPPB }\end{array}$ & $\begin{array}{l}\text { There was a statistically significant effect of the SG over the CG in } \\
\text { reducing fear of falling at week } 24 \text { (MFES difference }=0.85 \text { point, } 95 \% \text { CI } \\
0.26 \text { to } 1.44, p<0.01 \text { ) and reverse results in the case of KES (CG }>\text { SG) } \\
\text { (difference }=-6.8 \%, 95 \% \text { CI }-10.1 \text { to }-3.4, p<0.001) .\end{array}$ \\
\hline van den Berg M. et al. [31] & Mobility, risk of falling, balance & SPPB, MBR, Step Test, RMI, AM-PAC & $\begin{array}{l}\text { No impact on overall mobility. SG performed significantly better than CG } \\
\text { for MBR at Week } 2 \text { (between-group difference after baseline adjustment } \\
\qquad 38 \mathrm{~mm}, 95 \% \text { CI } 6 \text { to } 69 \text { ). }\end{array}$ \\
\hline Yeşilyaprak S.S. et al. [46] & Balance, fall risk & BBS, TUG, OLST, TS, FES-I & $\begin{array}{c}\text { Similar improvements were found in balance }(\text { BBS } p<0.01, \mathrm{~d}=0.43) \text { and } \\
\text { fall risk (TUG } p=0.01, \mathrm{~d}=0.41 \text { ) in both the SG and CG. There were no } \\
\text { differences between groups. }\end{array}$ \\
\hline Mirelman A. et al. [38] & $\begin{array}{l}\text { Gait analysis, balance, endurance, } \\
\text { risk of falling, physical activity }\end{array}$ & $\begin{array}{l}\text { Gait speed, leading foot clearance } \\
\text { from obstacle during walking, } \\
\text { 2MWT, SPPB, PASE }\end{array}$ & $\begin{array}{c}\text { Treadmill training plus VR led to reduced fall rates compared with } \\
\text { treadmill training alone for all participants (IRR } 0.58,95 \% \text { CI } 0.36-0.96 \text {; } \\
p=0.033 \text { ) and for participants with Parkinson's disease (RR } 0.45,95 \% \text { CI } \\
0.24-0.86 ; p=0.015 \text { ). However, incident rates after training did not differ } \\
\text { significantly between groups among people with idiopathic falls or } \\
\text { participants with mild cognitive impairment. }\end{array}$ \\
\hline Tsang W.W. et al. [35] & Balance, risk of falling & $\begin{array}{l}\text { BBS, TUG, stability test: } \\
\text {-reaction time } \\
\text {-end-point excursion } \\
\text {-maximum excursion } \\
\text {-stability directional control }\end{array}$ & $\begin{array}{l}\text { Significant improvement in balance was achieved in the SG in comparison } \\
\text { to CG, namely: BBS score }(p<0.001) \text {, reaction time in the anterior } \\
\text { direction }(p=0.003) \text {, end-point excursion, and maximum excursion in all } \\
\text { four directions of the limits of stability test }(p<0.001) \text {. }\end{array}$ \\
\hline Eggenberger P. et al. [32] & $\begin{array}{l}\text { Gait analysis, functional fitness, fall } \\
\text { frequencies, fear of falling }\end{array}$ & $\begin{array}{l}\text { GAITRite electronic walkway, SPPB, } \\
\text { 6-MWT, FES-I }\end{array}$ & $\begin{array}{l}\text { SG has a significant advantage over CG in the costs of step time variability } \\
\left.\text { in fast walking } \mathrm{F} 1,136=2.95, p=0.044 \text {, one-tailed, } \mathrm{R}^{2}=0.010\right) \text {. Global } \\
\text { linear time effects showed improved gait functional fitness and reduced } \\
\text { fall frequency without differences between interventions. }\end{array}$ \\
\hline Gschwind Y.J. et al. [47] & $\begin{array}{l}\text { Risk of falling, health measures, } \\
\text { physical measures }\end{array}$ & $\begin{array}{c}\text { PPA, WHODAS 2.0, Icon-FES, SPPB, } \\
\text { TUG }\end{array}$ & $\begin{array}{l}\text { Fall risk was significantly reduced in the SG compared with the CG } \\
(\mathrm{F} 1,127=4.54, p=0.035) \text {. Participants with higher adherence also } \\
\text { improved in postural sway (F1,75 }=4.13, p=0.046) \text {, stepping reaction } \\
(\mathrm{F} 1,75=4.40, p=0.041) \text {, and executive function }(\mathrm{F} 1,71=4.21, p=0.044) \text {. }\end{array}$ \\
\hline
\end{tabular}


Table 2. Cont.

\begin{tabular}{|c|c|c|c|}
\hline First Author & Outcomes & Research Tools & Results \\
\hline Gschwind Y.J. et al. [30] & $\begin{array}{l}\text { Risk of falling, functional mobility, } \\
\text { proprioception, reaction time, } \\
\text { muscle strength }\end{array}$ & WHODAS 2.0, PPA, TUG, STS & $\begin{array}{l}\text { SG reduced significantly in fall risk }(p=0.36) \text {, improved proprioception } \\
(p=0.015) \text {, and isometric knee extension strength }(p=0.32) \text { in comparison } \\
\text { to the CG. }\end{array}$ \\
\hline Jung D.I. et al. [37] & $\begin{array}{l}\text { Static balance, dynamic balance, } \\
\text { obstacle gait }\end{array}$ & BBS, FRT, TUG, CV, MVHC & $\begin{array}{l}\text { Both interventions-LSE and exergames-showed significant } \\
\text { improvements in obstacle negotiation function and falling-related balance } \\
\text { in elderly women at risk for falls (BBS, FRT, CV and TUG, MVHC } \\
\qquad p<0.001) .\end{array}$ \\
\hline Park J. et al. [48] & $\begin{array}{l}\text { Muscle strength, standing balance, } \\
\text { sitting balance }\end{array}$ & $\begin{array}{l}\text { Arm curl and handgrip strength } \\
\text { tests, Good Balance system }\end{array}$ & $\begin{array}{l}\text { Muscle strength and balance were significantly improved }(p<0.05) \text { in the } \\
\text { SG compared with the CG. }\end{array}$ \\
\hline Whyatt et. al. [36] & Balance, balance confidence & $\begin{array}{l}\text { BBS, NeuroCom Balance Master: } \\
\text { recordings of COP displacement }\end{array}$ & $\begin{array}{l}\text { Significant effect on levels of functional balance and balance confidence in } \\
\text { the SG compared to CG in BBS }\left(\mathrm{F} 1,79=3.640, p=0.06, \eta^{2}=0.044\right) \text {. The SG } \\
\text { also showed a significant effect on levels of dynamic postural control } \\
\text { relative to CG }\left(\mathrm{F} 1,80=39.54, p<0.001, \eta^{2}=0.331\right) .\end{array}$ \\
\hline \multicolumn{4}{|c|}{ 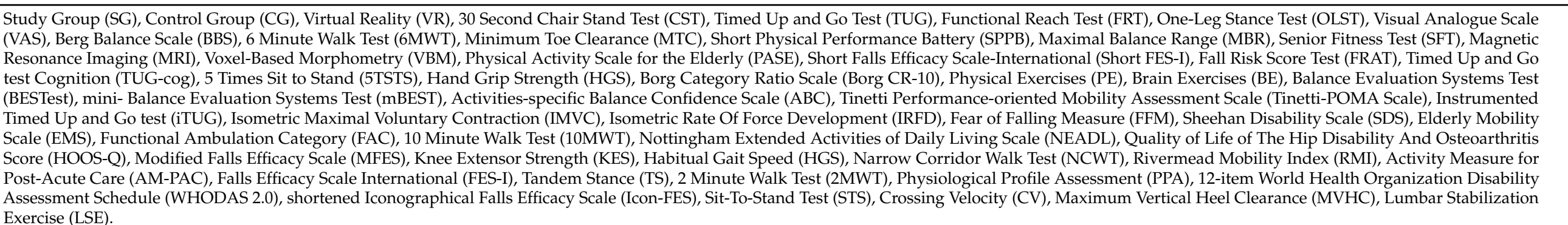 } \\
\hline
\end{tabular}




\subsubsection{Qualitative Outcomes}

Among the studies included, 14 of them $(63.6 \%)$ assessed qualitative variables such as cognitive function, quality of life, adherence, useability, and enjoyment of exergames. Table 3 presents detailed results of qualitative outcomes. It has been noted that the use of virtual reality rehabilitation is cost-effective, as compared to standard geriatric rehabilitation in the OTAGO system, due to the reduction of costs related to personnel work, their training, and community [42]. Additional virtual reality equipment does not significantly increase the costs associated with this type of therapy [38]. Most authors highlight the safety and absence of adverse effects in VR training for geriatric patients [31,33,34,39,40,42,47]. However, some adverse events were reported, such as pain during exercises [44], knee joint and tight soreness [45], or those unrelated directly to interventions [30]. Exergames were assessed as an acceptable and pleasant form of treatment in five out of seven studies. In five of nine studies, the VR rehabilitation significantly improved various cognitive functions among the elderly. An assessment of the quality of life among participants using VR training indicates that they mostly achieved greater improvement in QoL parameters than controls (four from five studies). This could be caused by the attractiveness of this kind of intervention. The conclusions formulated above suggest a positive [clear] influence of VR training on those qualitative variables. However, there was no influence of exergames on decreasing the symptoms of depression. This section may be divided by subheadings. It should provide a concise and precise description of the experimental results, their interpretation, as well as the experimental conclusions that can be drawn. 
Table 3. Qualitative outcomes.

\section{First Author}

Yang C.M. et al. [45]

Adcock M. et al. [39]

Stanmore E.K. et al. [42]

Liao Y. et al. [40]

Delbroek T. et al. [43]

Padala K.P. et al. [33]

Oesch P. et al. [44]

Levy F. et al. [29]

van den Berg M. et al. [31]

Mirelman A. et al. [38]

Eggenberger P. et al. [32]

Gschwind Y.J. et al. [47]

Gschwind Y.J. et al. [30]

Park J. et al. [48]

\section{Outcomes}

Feasibility and safety

Cognitive function

Cognitive function, depression, quality of life

Executive function

Cognition, motivation, emotions

Enjoyment of exercise, cognition, quality of life

Adherence, motivation and enjoyment

Fear of falling, depression, anxiety

Quality of life, feasibility, safety, usability, enjoyment

Cognition, health-related quality of life

$$
\text { Depression }
$$

Quality of life, depression, cognitive measures, executive function, adherence, usability

Cognitive, executive functioning Cognitive function

Research Tools

Results

Recording the participants' overall participation rate and attendance rate.

VST, TMT, WMS-R

ACEIII, 5-item GDS, HRQoL, Euro-QoL EQ-5D, SUS

TMT, SCWT

MOCA, IMI, OERS

PACES, 3MS, SF-36

daily training volume by standardized logbook with self-reported measuresfive-level Likert scale

BDI (21-item version), STAI-Y-A, STAI-Y-B

Euro-QoL EQ-5D, RNLI, SUS, PACES

EFI, AIS, SF-36

GDS

Euro-QoL EQ-5D, PHQ-9, VST, DSB, ANT, SUS, PACES, DART

Mini-Cog, ANT, VST, DSB

$\mathrm{MoCA}$
The Kinect exercise group had a 100\% overall participation rate, with all participants attending the entire training.

No significant difference between the study and control group in cognitive function. Significant improvement in executive functioning (working memory and inhibition). No significant difference between the study and control group in cognitive function, depression, and quality of life. Exergames had high acceptance, usability, and feasibility. Only VR training improved cognitive dual-task gait.

No significant improvement in cognitive function compared to control. VR training is a pleasant and acceptable treatment for older adults.

No significant improvement in cognition, quality of life, and high enjoyment in VR training group in comparison to controls.

The adherence, motivation, and enjoyment were significantly higher in standard exercise training than exergames.

No significant difference between groups in depression, trait anxiety, or improvement in VR group compared to controls only in the case of state anxiety.

Participants were comfortable with the technology and that the equipment was easy to use and enjoyed the intervention. Significant improvement of quality of life in VR group compared controls.

Cognitive function outcomes improved similarly in both training groups. However, the quality of life improved more in VR group.
No significant difference between groups.

Significantly higher adherence, improvement in quality of life, and executive functioning were found in VR group in comparison controls. The intervention was enjoyed by the participants, and its usability was acceptable.

The study group improved specific cognitive functions, finger-press reaction time, Stroop time compared to controls.

Cognitive function was significantly improved in the study group compared to the

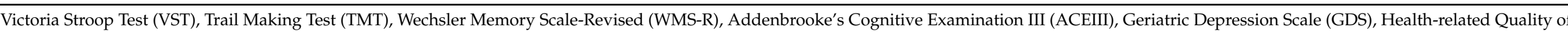

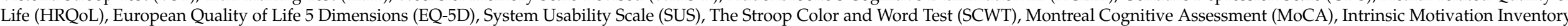

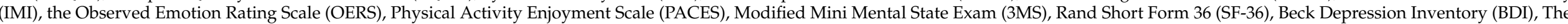

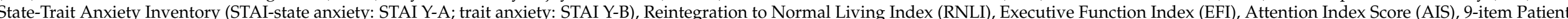
Health Questionnaire (PHQ-9), Victoria Stroop Test (VST), Digit Span Backward (DSB), Attention Network Test (ANT), Dynamic Acceptance Model for the Re-evaluation of Technologies (DART). 


\subsection{Quality Assessment}

The PEDro scale was used to evaluate the methodological quality of the included studies (Table 4). Among the 21 studies, 4 out of $21(19 \%)$ were of high quality (10-7 points) and 17 out of $21(81 \%)$ were of moderate quality (6-4 points). The average quality rating of the analyzed articles was 5.8 points (moderate quality). The main sources of bias were the lack of blindness for participants and therapists (all studies), which was impossible to obtain for this intervention, and the lack of application of intention to treat data analysis (only 7 from 21, 33.3\%).

Table 4. The methodological quality assessment of the articles included in PEDro Scale.

\begin{tabular}{ccccccccccccc}
\hline First Author & EC & RA & CA & BC & BS & BT & BA & AF & ITA & BGC & PEaV & Total Score \\
\hline Yang C.M. et al. [45] & + & + & - & + & - & - & + & + & - & + & + & $6 / 10$ \\
Ehrari H. et al. [28] & + & + & + & + & - & - & + & - & - & + & + & $6 / 10$ \\
Adcock M. et al. [39] & + & + & + & + & - & - & - & - & - & + & + & $5 / 10$ \\
Stanmore E.K. et al. [42] & + & + & - & + & - & - & - & + & + & + & + & $6 / 10$ \\
Liao Y. et al. [40] & + & + & + & + & - & - & + & - & - & + & + & $6 / 10$ \\
Htut T. et al. [41] & + & + & - & + & - & - & + & + & - & + & + & $6 / 10$ \\
Delbroek T. et al. [43] & + & + & - & + & - & - & + & - & - & - & + & $4 / 10$ \\
Padala K.P. et al. [33] & + & + & + & + & - & - & - & + & + & + & + & $7 / 10$ \\
Oesch P. et al. [44] & + & + & - & + & - & - & - & - & + & + & + & $5 / 10$ \\
Levy F. et al. [29] & + & - & - & + & - & - & + & + & + & + & + & $6 / 10$ \\
Kwok B.C. et al. [34] & + & + & + & + & - & - & + & - & - & + & + & \\
van den Berg M. et al. [31] & + & + & + & + & - & - & + & + & - & + & + & $7 / 10$ \\
Yesilyaprak S.S. et al. [46] & + & + & - & + & - & - & + & + & - & + & + & $6 / 10$ \\
Mirelman A. et al. [38] & + & + & + & + & - & - & + & + & + & + & + & $8 / 10$ \\
Tsang W.W. et al. [35] & + & + & - & + & - & - & - & + & + & + & + & $6 / 10$ \\
Eggenberger P. et al. [32] & + & + & - & + & - & - & - & - & - & + & + & $4 / 10$ \\
Gschwind Y.J. et al. [47] & - & + & - & + & - & - & - & + & + & + & + & $6 / 10$ \\
Gschwind Y.J. et al. [30] & + & + & + & + & - & - & + & - & + & + & + & $7 / 10$ \\
Jung D.I. et al. [37] & + & + & - & + & - & - & - & - & - & + & + & $4 / 10$ \\
Park J. et al. [48] & + & + & - & + & - & - & - & + & - & + & + & $5 / 10$ \\
Whyatt et. al. [36] & + & + & - & + & - & - & - & + & - & + & + & $5 / 10$ \\
\hline
\end{tabular}

* Eligibility criteria it is not included in the total score; EC, Eligibility criteria; RA, Random allocation; CA, Concealed allocation; BC, Baseline comparability; BS, Blind subjects; BT, Blind therapists; BA, Blind assessors; AF, Adequate follow-up; ITA, Intention-to-treat analysis; BGC, Between-group comparisons; PEaV, Point Estimates and Variability; (1 point: “+"; 0 point: “-").

\section{Discussion}

The aim of the present review was to determine the effectiveness of a rehabilitation accommodating virtual reality and exergames in view of fall prevention among elderly people.

The progress of technology resulted in the utilization of virtual reality in medical appliances [49]. Virtual reality techniques have been widely adopted in other fields of medicine, including specialist training like simulations of surgical procedures [50], pain management [51,52], treatment of phobias or anxiety [53], and diagnostics [54]. Growing interest regarding virtual reality appliances and a wide variety of available software applications and hardware devices resulted in wider adoption in rehabilitation in view of making the rehabilitation more attractive, reducing total costs related to human and infrastructural factors (telerehabilitation), as well as considering maximizing the effects of traditional physiotherapeutic proceedings.

Limitations of motor and cognitive functions, aggravating with age, result in a vast representation of elderly people among patients who are a subject of rehabilitation [55]. Nowadays, virtual reality rehabilitation is considered as an intervention targeted at children [56], adolescents and adults [57], athletes [58], as well as the elderly. However, varying motivation was observed among the age groups-children and adolescents are motivated by a challenging part of exergames like competition and gaining rewards/points in a game. 
On the other hand, older adults focus on perceived health benefits and enjoyment without competition [59]. Similarly, gaming was perceived as a domain of young people. Besides, one should remember that our contemporary digital world was created by a generation of people, who are currently in their 50s. Recent studies have proved that older people draw substantial satisfaction and present good acceptance of virtual reality applications in the process of rehabilitation [33,42-45,47].

Moreover, virtual reality gives many opportunities to provide patient rehabilitation. It appears as a valuable rehabilitation tool regarding older people used in nursing homes, activity centers for the elderly, hospitals, and rehabilitation clinics, where qualified staff take care of the proper adoption and safety of interventions. The development of modern technology allows using VR rehabilitation in patients' homes successfully by means of telerehabilitation $[39,42,47]$. Telerehabilitation increases motivation to home exercises and continuity of rehabilitation after discharge from the hospital $[60,61]$. This is especially important during the coronavirus pandemic where many people must stay at home and access to indirect rehabilitation is limited. Additionally, VR rehabilitation seems to be effective in reducing the cost and time of personal staff and the daily commute to the health center [42]. However, most previous implementations relied on telerehabilitation, hence there is only scarce evidence of virtual reality in this field.

This review suggests that virtual reality training in rehabilitation appears to be promising complementation or even an alternative to traditional techniques of physiotherapy. However, several factors, which can limit its wider application among elderly people, should be considered. Seniors are frequently reluctant in unassisted habituation of remote rehabilitation, which could be partially explained by still cursory knowledge of modern technologies. Such a situation can be solved by the implementation of possibly simple systems, adopted to requirements and capabilities of elderly people, provided with capabilities to reversely monitor the activity of patients, proper execution of exercises, and supporting mechanisms of motivation.

Regarding the quantitative outcomes of this review, the question of whether virtual reality offers greater effectiveness over traditional rehabilitation shifts towards a positive answer. The analysis of studies included in this review suggests that rehabilitation accommodating virtual reality, aimed at improving physical functions, including body balance, gait, and muscular strength, has some effectiveness. However, due to differences in applied technology and protocols, it is difficult to draw clear conclusions. These findings are in compliance with the research works of other authors. Pacheco et al. [12] found exergames to improve balance and mobility in older adults with impairments without neurological diseases. Accommodation of those elements of motor activity in exergames in the rehabilitation resulted in an improvement of the body balance among elderly people [62]; however, the significant effect was related to the dynamic and perceived balance, but not to the static balance.

Several studies show the benefits of tai-chi, yoga, and rehabilitation exercises programs regarding the decreased risk of falls among elderly people [63-65]. Although their effectiveness is undebatable, elderly patients currently need remote intervention to keep their mobility safe. Therefore, virtual reality rehabilitation and exergames begin playing a role in telerehabilitation, and as is proved in this review, they constitute an equally as effective form of intervention as traditional treatment in view of mobility and balance improvement, as well as reduction of the risk of falls.

An important factor of rehabilitation of elderly people, which should be considered, is a deterioration of cognitive functions during aging. This review mostly found exergames to be an acceptable and pleasant treatment. In most studies, exergames significantly improved various cognitive functions, as well as the quality of life among elderly with a risk of falling.

Benefits regarding the health-related quality of life were also observed in elderly people who underwent virtual reality training. The level of satisfaction and enjoyment of such interventions was appraised positively [66]. There is also evidence of the positive motivational aspect of exergames among older adults [67]. 
In view of these qualitative outcomes, only a few authors have reported adverse effects, which were identified chiefly as non-related to intervention [38,44,45]. Incidentally, the comparison of adverse effects during VR rehabilitation in relation to traditional rehabilitation builds up another interesting research question.

Although exergames were assessed as an effective intervention in alleviating symptoms of depression [68], the studies in this review do not confirm this thesis. However, differences in the results could be caused by the participants' level of functional abilitiesthere were elderly with decreased mobility; number of sessions- the duration was long; and the games' profile was centered around rehabilitation and functional performance, not enjoyment. Li et al. [68] indicated that the influence of exergames on depression is more effective in patients with relatively good mobility who play highly playful games in small numbers of sessions.

Contemporary literature reviews regarding the effectiveness of virtual reality in orthopedic rehabilitation suggest that the variety of devices used, the variety of their functions, and a lack of coherence in applied protocols result are significant problems related to the comparison of the acquired results [69]. The analysis of the papers included in the current review also varied regarding the period of the intervention, adopted devices, and trained functions. A multitude of applied interventions and equipment, as well as a variety of tools and methods of evaluation of the effectiveness of those interventions, make it difficult to conduct a metanalysis.

Conclusions of the analyzed studies do not allow to draw up any precise clinical guidelines regarding the duration, frequency, types of devices, and software implementations, as well as particular ailments and diseases in which virtual rehabilitation and exergames should be indicated.

This review has some limitations because it concerned only randomized controlled trials published in the last 5 years in English. The reason is that RCTs are considered a gold standard in medical sciences and give reliable information about the effectiveness of the applied intervention [70]. In the last decade, there has been a rapid growth of VR clinical application papers published in PubMed [71]. Also, the quick development of modern technology meant that only the newest papers gave the opportunity to assess the current state of art in this topic. Therefore, there is a risk that other valuable reports would not be included in this review.

In many studies, only clinical tests were used to assess physical outcomes, which provide valid results, but are fraught with a risk of bias due to human mistakes. Regarding those factors, future studies should use standardized biomechanical methods of assessment based on objective diagnostics tools.

Common limitations of the included trials were the small sample size $[29,33,37,43,45,46]$ and the lack of a long-term follow-up (6/21 studies with follow-up), as well as no blindness among participants and therapists (all studies). However, blindness in this kind of intervention is difficult to achieve but long-term follow-up and a proper study sample size are necessary to obtain reliable results.

Exergames can be considered as an evolution to virtual reality, and vice versa. Virtual reality techniques allow deeper immersion during training. Exergames involve better interest in training [5]. Besides, it is interesting to assess whether virtual reality and gaming simply add to interest and immersion or whether they act in a supra-addition.

The current review supports the hypothesis that virtual reality and exergame application among elderly people can positively influence physical functions, as well as cognitive functions of seniors. However, virtual reality and exergame rehabilitation were obligatorily implemented in parallel with traditional rehabilitation and thus are mostly considered as a complement of standard physiotherapy [11,43], as well as its continuation at home $[35,36,45]$. 


\section{Conclusions}

There is an evidence that interventions based on virtual reality and exergames are effective in improving specific physical and psychological outcomes among elderly.

Interventions based on virtual reality and exergames are promising in view of multifactor management, regarding motor and cognitive functions, as well as the quality of life and enjoyment. The use of virtual reality is cost-effective, safe, and bereft of adverse effects. Future research studies, notably randomized controlled trials, with well-defined research protocol, are desirable to determine the effectiveness of virtual reality in fall prevention.

Author Contributions: Conceptualization, J.P. and K.C.; methodology, J.P.; formal analysis, J.P. and K.C.; investigation, J.P. and K.C.; writing-original draft preparation, J.P. and K.C.; writing-review and editing, J.P. and K.C.; visualization, J.P.; supervision, K.C.; project administration, J.P.; funding acquisition, J.P. Both authors have read and agreed to the published version of the manuscript.

Funding: This research received no external funding. English editing was co-financed by the Ph.D. Candidates Society of the Jagiellonian University.

Conflicts of Interest: The authors declare no conflict of interest.

\section{References}

1. World Health Organization. WHO Global Report on Falls Prevention in Older Age; World Health Organization: Geneva, Switzerland, 2007; ISBN 9789241563.

2. Slade, S.C.; Carey, D.L.; Hill, A.-M.; Morris, M.E. Effects of falls prevention interventions on falls outcomes for hospitalised adults: Protocol for a systematic review with meta-analysis. BMJ Open 2017, 7, e017864. [CrossRef]

3. Khow, K.S.; Visvanathan, R. Falls in the Aging Population. Clin. Geriatr. Med. 2017, 33, 357-368. [CrossRef] [PubMed]

4. Chang, J.T.; Morton, S.C.; Rubenstein, L.Z.; Mojica, W.A.; Maglione, M.; Suttorp, M.J.; Roth, E.A.; Shekelle, P.G. Interventions for the prevention of falls in older adults: Systematic review and meta-analysis of randomised clinical trials. BMJ 2004, 328, 680 . [CrossRef] [PubMed]

5. Perez-Marcos, D. Virtual reality experiences, embodiment, videogames and their dimensions in neurorehabilitation. J. Neuroeng. Rehabil. 2018, 15, 113. [CrossRef]

6. Paszkiel, S. Augmented Reality (AR) Technology in Correlation with Brain-Computer Interface Technology Immersion. In Studies in Computational Intelligence 852 Analysis and Classification of EEG Signals for Brain-Computer Interfaces; Springer: Cham, Switzerland, 2020; pp. 87-91. [CrossRef]

7. Paszkiel, S. Using BCI and VR Technology in Neurogaming. In Studies in Computational Intelligence 852 Analysis and Classification of EEG Signals for Brain-Computer Interfaces; Springer: Cham, Switzerland, 2020; pp. 93-99. [CrossRef]

8. Musk, E. An Integrated Brain-Machine Interface Platform with Thousands of Channels. J. Med. Internet Res. 2019, 21, e16194. [CrossRef] [PubMed]

9. Monteiro-Junior, R.S.; Vaghetti, C.A.O.; Nascimento, O.J.M.; Laks, J.; Deslandes, A.C. Exergames: Neuroplastic hypothesis about cognitive improvement and biological effects on physical function of institutionalized older persons. Neural Regen. Res. 2016, 11, 201-204. [CrossRef]

10. Costa, M.T.S.; Vieira, L.P.; Barbosa, E.D.O.; Oliveira, L.M.; Maillot, P.; Vaghetti, C.A.O.; Carta, M.G.; Machado, S.; Gatica-Rojas, V.; Monteiro-Junior, R.S. Virtual Reality-Based Exercise with Exergames as Medicine in Different Contexts: A Short Review. Clin. Pract. Epidemiol. Ment. Health 2019, 15, 15-20. [CrossRef]

11. Skjæret, N.; Nawaz, A.; Morat, T.; Schoene, D.; Helbostad, J.L.; Vereijken, B. Exercise and rehabilitation delivered through exergames in older adults: An integrative review of technologies, safety and efficacy. Int. J. Med. Inf. 2016, 85, 1-16. [CrossRef] [PubMed]

12. Pacheco, T.B.F.; De Medeiros, C.S.P.; De Oliveira, V.H.B.; Vieira, E.R.; De Cavalcanti, F.A.C. Effectiveness of exergames for improving mobility and balance in older adults: A systematic review and meta-analysis. Syst. Rev. 2020, 9, 1-14. [CrossRef] [PubMed]

13. Keil, J.; Edler, D.; O'Meara, D.; Korte, A.; Dickmann, F. Effects of Virtual Reality Locomotion Techniques on Distance Estimations. ISPRS Int. J. Geo-Inf. 2021, 10, 150. [CrossRef]

14. Syed-Abdul, S.; Malwade, S.; Nursetyo, A.A.; Sood, M.; Bhatia, M.; Barsasella, D.; Liu, M.F.; Chang, C.-C.; Srinivasan, K.; Raja, M.; et al. Virtual reality among the elderly: A usefulness and acceptance study from Taiwan. BMC Geriatr. 2019, 19, 1-10. [CrossRef]

15. Szpak, A.; Michalski, S.C.; Saredakis, D.; Chen, C.S.; Loetscher, T. Beyond Feeling Sick: The Visual and Cognitive Aftereffects of Virtual Reality. IEEE Access 2019, 7, 130883-130892. [CrossRef]

16. Champney, R.K.; Stanney, K.M.; Hash, P.A.K.; Malone, L.C.; Kennedy, R.S.; Compton, D.E. Recovery from virtual environment exposure: Expected time course of symptoms and potential readaptation strategies. Hum. Factors J. Hum. Factors Ergon. Soc. 2007, 49, 491-506. [CrossRef] [PubMed] 
17. Wright, W.G. Using virtual reality to augment perception, enhance sensorimotor adaptation, and change our minds. Front. Syst. Neurosci. 2014, 8, 56. [CrossRef] [PubMed]

18. Szpak, A.; Michalski, S.C.; Loetscher, T. Exergaming with Beat Saber: An Investigation of Virtual Reality Aftereffects. J. Med. Internet Res. 2020, 22, e19840. [CrossRef] [PubMed]

19. Ozdogar, A.T.; Ertekin, O.; Kahraman, T.; Yigit, P.; Ozakbas, S. Effect of video-based exergaming on arm and cognitive function in persons with multiple sclerosis: A randomized controlled trial. Mult. Scler. Relat. Disord. 2020, 40, 101966. [CrossRef] [PubMed]

20. Fiani, B.; Siddiqi, I.; Lee, S.C.; Dhillon, L. Telerehabilitation: Development, Application, and Need for Increased Usage in the COVID-19 Era for Patients with Spinal Pathology. Cureus 2020, 12, 10563. [CrossRef] [PubMed]

21. Wang, S.S.Y.; Teo, W.Z.W.; Teo, W.Z.Y.; Chai, Y.W. Virtual Reality as a Bridge in Palliative Care during COVID-19. J. Palliat. Med. 2020, 23, 756. [CrossRef]

22. Moher, D.; Liberati, A.; Tetzlaff, J.; Altman, D.G. The PRISMA Group. Preferred reporting items for systematic reviews and meta-analyses: The PRISMA statement. PLoS Med. 2009, 6, e1000097. [CrossRef]

23. Riva, J.J.; Malik, K.M.; Burnie, S.J.; Endicott, A.R.; Busse, J.W. What is your research question? An introduction to the PICOT format for clinicians. J. Can. Chiropr. Assoc. 2012, 56, 167-171.

24. Ouzzani, M.; Hammady, H.; Fedorowicz, Z.; Elmagarmid, A. Rayyan-A web and mobile app for systematic reviews. Syst. Rev. 2016, 5, 1-10. [CrossRef]

25. Maher, C.G.; Sherrington, C.; Herbert, R.D.; Moseley, A.M.; Elkins, M. Reliability of the PEDro Scale for Rating Quality of Randomized Controlled Trials. Phys. Ther. 2003, 83, 713-721. [CrossRef] [PubMed]

26. Olivo, S.A.; Macedo, L.G.; Gadotti, I.C.; Fuentes, J.; Stanton, T.; Magee, D.J. Scales to Assess the Quality of Randomized Controlled Trials: A Systematic Review. Phys. Ther. 2008, 88, 156-175. [CrossRef] [PubMed]

27. PEDro Scale. Available online: https:/ / www.pedro.org.au/wp-content/uploads/PEDro_scale.pdf (accessed on 5 January 2021).

28. Ehrari, H.; Larsen, R.T.; Langberg, H.; Andersen, H.B. Effects of Playful Exercise of Older Adults on Balance and Physical Activity: A Randomized Controlled Trial. J. Popul. Ageing 2020, 13, 207-222. [CrossRef]

29. Levy, F.; Rautureau, G.; Komano, O.; Millet, B.; Jouvent, R.; Leboucher, P. Fear of falling: Efficacy of virtual reality associated with serious games in elderly people. Neuropsychiatr. Dis. Treat. 2016, 12, 877-881. [CrossRef]

30. Gschwind, Y.J.; Schoene, D.; Lord, S.R.; Ejupi, A.; Valenzuela, T.; Aal, K.; Woodbury, A.; Delbaere, K. The effect of sensorbased exercise at home on functional performance associated with fall risk in older people-A comparison of two exergame interventions. Eur. Rev. Aging Phys. Act. 2015, 12, 11. [CrossRef]

31. Berg, M.V.D.; Sherrington, C.; Killington, M.; Smith, S.T.; Bongers, B.; Hassett, L.; Crotty, M. Video and computer-based interactive exercises are safe and improve task-specific balance in geriatric and neurological rehabilitation: A randomised trial. J. Physiother. 2016, 62, 20-28. [CrossRef]

32. Eggenberger, P.; Theill, N.; Holenstein, S.; Schumacher, V.; De Bruin, E.D. Multicomponent physical exercise with simultaneous cognitive training to enhance dual-task walking of older adults: A secondary analysis of a 6-month randomized controlled trial with 1-year follow-up. Clin. Interv. Aging 2015, 10, 1711-1732. [CrossRef]

33. Padala, K.P.; Padala, P.R.; Lensing, S.Y.; Dennis, R.A.; Bopp, M.M.; Parkes, C.M.; Garrison, M.K.; Dubbert, P.M.; Roberson, P.K.; Sullivan, D.H. Efficacy of Wii-Fit on Static and Dynamic Balance in Community Dwelling Older Veterans: A Randomized Controlled Pilot Trial. J. Aging Res. 2017, 2017, 1-9. [CrossRef]

34. Kwok, B.C.; Pua, Y.H. Effects of WiiActive exercises on fear of falling and functional outcomes in community-dwelling older adults: A randomised control trial. Age Ageing 2016, 45, 621-627. [CrossRef]

35. Tsang, W.W.; Fu, A.S. Virtual reality exercise to improve balance control in older adults at risk of falling. Hong Kong Med. J. 2016, 22 (Suppl. 2), S19-S22. [CrossRef]

36. Whyatt, C.; Merriman, N.A.; Young, W.R.; Newell, F.N.; Craig, C. A Wii Bit of Fun: A Novel Platform to Deliver Effective Balance Training to Older Adults. Games Health J. 2015, 4, 423-433. [CrossRef]

37. Jung, D.-I.; Ko, D.-S.; Jeong, M.-A. Kinematic effect of Nintendo WiiTM sports program exercise on obstacle gait in elderly women with falling risk. J. Phys. Ther. Sci. 2015, 27, 1397-1400. [CrossRef] [PubMed]

38. Mirelman, A.; Rochester, L.; Maidan, I.; Del Din, S.; Alcock, L.; Nieuwhof, F.; Rikkert, M.O.; Bloem, B.R.; Pelosin, E.; Avanzino, L.; et al. Addition of a non-immersive virtual reality component to treadmill training to reduce fall risk in older adults (V-TIME): A randomised controlled trial. Lancet 2016, 388, 1170-1182. [CrossRef]

39. Adcock, M.; Fankhauser, M.; Post, J.; Lutz, K.; Zizlsperger, L.; Luft, A.R.; Guimarães, V.; Schättin, A.; De Bruin, E.D. Effects of an In-home Multicomponent Exergame Training on Physical Functions, Cognition, and Brain Volume of Older Adults: A Randomized Controlled Trial. Front. Med. 2020, 6, 321. [CrossRef] [PubMed]

40. Liao, Y.-Y.; Chen, I.-H.; Lin, Y.-J.; Chen, Y.; Hsu, W.-C. Effects of Virtual Reality-Based Physical and Cognitive Training on Executive Function and Dual-Task Gait Performance in Older Adults with Mild Cognitive Impairment: A Randomized Control Trial. Front. Aging Neurosci. 2019, 11, 162. [CrossRef]

41. Htut, T.Z.C.; Hiengkaew, V.; Jalayondeja, C.; Vongsirinavarat, M. Effects of physical, virtual reality-based, and brain exercise on physical, cognition, and preference in older persons: A randomized controlled trial. Eur. Rev. Aging Phys. Act. 2018, 15, 1-12. [CrossRef] [PubMed] 
42. Stanmore, E.K.; Mavroeidi, A.; De Jong, L.D.; Skelton, D.A.; Sutton, C.J.; Benedetto, V.; Munford, L.A.; Meekes, W.; Bell, V.; Todd, $\mathrm{C}$. The effectiveness and cost-effectiveness of strength and balance Exergames to reduce falls risk for people aged 55 years and older in UK assisted living facilities: A multi-centre, cluster randomised controlled trial. BMC Med. 2019, 17, 1-14. [CrossRef] [PubMed]

43. Delbroek, T.; Vermeylen, W.; Spildooren, J. The effect of cognitive-motor dual task training with the biorescue force platform on cognition, balance and dual task performance in institutionalized older adults: A randomized controlled trial. J. Phys. Ther. Sci. 2017, 29, 1137-1143. [CrossRef]

44. Oesch, P.; Kool, J.; Fernandez-Luque, L.; Brox, E.; Evertsen, G.; Civit, A.; Hilfiker, R.; Bachmann, S. Exergames versus self-regulated exercises with instruction leaflets to improve adherence during geriatric rehabilitation: A randomized controlled trial. $B M C$ Geriatr. 2017, 17, 77. [CrossRef]

45. Yang, C.M.; Chen Hsieh, J.S.; Chen, Y.C.; Yang, S.Y.; Lin, H.C.K. Effects of Kinect exergames on balance training among community older adults: A randomized controlled trial. Medicine 2020, 99, e21228. [CrossRef] [PubMed]

46. Yeşilyaprak, S.S.; Yıldırım, M. Şenduran; Tomruk, M.; Ertekin, Özge; Algun, Z.C. Comparison of the effects of virtual reality-based balance exercises and conventional exercises on balance and fall risk in older adults living in nursing homes in Turkey. Physiother. Theory Pract. 2016, 32, 191-201. [CrossRef]

47. Gschwind, Y.J.; Eichberg, S.; Ejupi, A.; De Rosario, H.; Kroll, M.; Marston, H.R.; Drobics, M.; Annegarn, J.; Wieching, R.; Lord, S.R.; et al. ICT-based system to predict and prevent falls (iStoppFalls): Results from an international multicenter randomized controlled trial. Eur. Rev. Aging Phys. Act. 2015, 12, 1-11. [CrossRef] [PubMed]

48. Park, J.; Yim, J. A New Approach to Improve Cognition, Muscle Strength, and Postural Balance in Community-Dwelling Elderly with a 3-D Virtual Reality Kayak Program. Tohoku J. Exp. Med. 2016, 238, 1-8. [CrossRef] [PubMed]

49. Riener, R.; Harders, M. Virtual Reality in Medicine; Springer: London, UK, 2012. [CrossRef]

50. Vaughan, N.; Dubey, V.N.; Wainwright, T.W.; Middleton, R.G. A review of virtual reality based training simulators for orthopaedic surgery. Med. Eng. Phys. 2016, 38, 59-71. [CrossRef]

51. Rutledge, T.; Velez, D.; Depp, C.; McQuaid, J.R.; Wong, G.; Jones, R.C.W.; Atkinson, J.H.; Giap, B.; Quan, A.; Giap, H. A Virtual Reality Intervention for the Treatment of Phantom Limb Pain: Development and Feasibility Results. Pain Med. 2019, 20, $2051-2059$. [CrossRef]

52. Tashjian, V.C.; Mosadeghi, S.; Howard, A.R.; Lopez, M.; Dupuy, T.; Reid, M.; Martinez, B.; Ahmed, S.; Dailey, F.; Robbins, K.; et al. Virtual Reality for Management of Pain in Hospitalized Patients: Results of a Controlled Trial. JMIR Ment. Health 2017, 4, e9. [CrossRef]

53. Lindner, P.; Miloff, A.; Hamilton, W.; Reuterskiöld, L.; Andersson, G.; Powers, M.B.; Carlbring, P. Creating state of the art, nextgeneration Virtual Reality exposure therapies for anxiety disorders using consumer hardware platforms: Design considerations and future directions. Cogn. Behav. Ther. 2017, 46, 404-420. [CrossRef]

54. Negrillo-Cárdenas, J.; Jiménez-Pérez, J.-R.; Feito, F.R. The role of virtual and augmented reality in orthopedic trauma surgery: From diagnosis to rehabilitation. Comput. Methods Programs Biomed. 2020, 191, 105407. [CrossRef]

55. Galloza, J.; Castillo, B.; Micheo, W. Benefits of Exercise in the Older Population. Phys. Med. Rehabil. Clin. N. Am. 2017, 28, 659-669. [CrossRef]

56. Meyns, P.; Pans, L.; Plasmans, K.; Heyrman, L.; Desloovere, K.; Molenaers, G. The Effect of Additional Virtual Reality Training on Balance in Children with Cerebral Palsy after Lower Limb Surgery: A Feasibility Study. Games Health J. 2017, 6, 39-48. [CrossRef] [PubMed]

57. Silva, V.; Campos, C.; Sá, A.; Cavadas, M.; Pinto, J.; Simões, P.; Machado, S.; Murillo-Rodríguez, E.; Barbosa-Rocha, N. Wii-based exercise program to improve physical fitness, motor proficiency and functional mobility in adults with Down syndrome. $J$. Intellect. Disabil. Res. 2017, 61, 755-765. [CrossRef]

58. Chung, L.M.Y.; Sun, F.H.; Cheng, C.T.M.; Sun, B.F. Physiological and Perceived Responses in Different Levels of Exergames in Elite Athletes. Games Health J. 2017, 6, 57-60. [CrossRef]

59. Subramanian, S.; Dahl, Y.; Maroni, N.S.; Vereijken, B.; Svanæs, D. Assessing Motivational Differences between Young and Older Adults When Playing an Exergame. Games Health J. 2020, 9, 24-30. [CrossRef] [PubMed]

60. Wang, X.; Hunter, D.J.; Vesentini, G.; Pozzobon, D.; Ferreira, M.L. Technology-assisted rehabilitation following total knee or hip replacement for people with osteoarthritis: A systematic review and meta-analysis. BMC Musculoskelet. Disord. 2019, $20,1-17$. [CrossRef] [PubMed]

61. Pastora-Bernal, J.M.; Martín-Valero, R.; Barón-López, F.J.; Estebanez-Pérez, M.J. Evidence of benefit of telerehabitation after orthopedic surgery: A systematic review. J. Med. Internet Res. 2017, 19, e142. [CrossRef] [PubMed]

62. Fang, Q.; Ghanouni, P.; Anderson, S.E.; Touchett, H.; Shirley, R.; Fang, F.; Fang, C. Effects of Exergaming on Balance of Healthy Older Adults: A Systematic Review and Meta-analysis of Randomized Controlled Trials. Games Health J. 2020, 9, 11-23. [CrossRef]

63. Saravanakumar, P.; Higgins, I.J.; Van Der Riet, P.J.; Marquez, J.; Sibbritt, D. The influence of tai chi and yoga on balance and falls in a residential care setting: A randomised controlled trial. Contemp. Nurse 2014, 48, 76-87. [CrossRef]

64. Huang, Z.-G.; Feng, Y.-H.; Li, Y.-H.; Lv, C.-S. Systematic review and meta-analysis: Tai Chi for preventing falls in older adults. BMJ Open 2017, 7, e013661. [CrossRef]

65. Beato, M.; Dawson, N.; Svien, L.; Wharton, T. Examining the Effects of an Otago-Based Home Exercise Program on Falls and Fall Risks in an Assisted Living Facility. J. Geriatr. Phys. Ther. 2019, 42, 224-229. [CrossRef] 
66. Cacciata, M.; Stromberg, A.; Lee, J.-A.; Sorkin, D.; Lombardo, D.; Clancy, S.; Nyamathi, A.; Evangelista, L.S. Effect of exergaming on health-related quality of life in older adults: A systematic review. Int. J. Nurs. Stud. 2019, 93, 30-40. [CrossRef] [PubMed]

67. Molina, K.I.; Ricci, N.A.; De Moraes, S.A.; Perracini, M.R. Virtual reality using games for improving physical functioning in older adults: A systematic review. J. Neuroeng. Rehabil. 2014, 11, 156. [CrossRef] [PubMed]

68. Li, J.; Theng, Y.-L.; Foo, S. Effect of Exergames on Depression: A Systematic Review and Meta-Analysis. Cyberpsychol. Behav. Soc. Netw. 2016, 19, 34-42. [CrossRef]

69. Byra, J.; Czernicki, K. The Effectiveness of Virtual Reality Rehabilitation in Patients with Knee and Hip Osteoarthritis. J. Clin. Med. 2020, 9, 2639. [CrossRef] [PubMed]

70. Moher, D.; Hopewell, S.; Schulz, K.F.; Montori, V.; Gøtzsche, P.C.; Devereaux, P.J.; Elbourne, D.; Egger, M.; Altman, D.G. CONSORT 2010 explanation and elaboration: Updated guidelines for reporting parallel group randomised trials. BMJ 2010, 340, c869. [CrossRef] [PubMed]

71. Garrett, B.; Taverner, T.; Gromala, D.; Tao, G.; Cordingley, E.; Sun, C. Virtual Reality Clinical Research: Promises and Challenges. JMIR Serious Games 2018, 6, e10839. [CrossRef] [PubMed] 